\title{
A Survey of Avifauna in Bachok District, Kelantan, Peninsular Malaysia.
}

\author{
Ramli R.*, Ya'cob Z., Aimi F. and N.H. Ezyan \\ Institute of Biological Sciences, Faculty of Science, University of Malaya, 50603 Kuala Lumpur, Malaysia. \\ * rosliramli@um.edu.my (corresponding author) \\ Received on $22^{\text {nd }}$ June 2009, accepted in revised form $30^{\text {th }}$ September 2009.
}

\begin{abstract}
A survey on bird diversity was conducted along coastal areas of Bachok, Kelantan. The aim of the study is to document avian species diversity in the proposed site for UM Marine Field Station. Field visits were conducted five times over a period of 12 months from June 2008 until May 2009. The direct observation technique was applied to record bird species. A total of 70 species of birds from 30 families were recorded in the study area. Of this, three species were introduced species, 45 were residents, 11 were migrants, and 11 species have both migrants and residents populations. Forty-eight of the recorded species are either totally protected or partially protected (two species as game birds and other two species as licence caged birds) under Protection of Wildlife Act 1976. In term of conservation status, two species (Grey-breasted Babbler, Malacopteron albogulare and Longtailed Parakeet, Psittacula longicauda) are classified as Nearly Threatened by the IUCN Red Data Book. Fifty-two recorded species are commonly found throughout Peninsular Malaysia but ten species are uncommon, seven are abundant, and a single species (Javan Pond-Heron, Ardeola speciosa) is rarely found. Bird distributions and species diversity recorded during each visit varied depending on sampling site. Generally, Bachok district possesses a wide variety of birds and can contribute significantly towards biodiversity conservation and management.
\end{abstract}

ABSTRAK Satu bancian ke atas kepelbagaian spesies burung telah dijalankan di sepanjang kawasan pantai di Bachok, Kelantan. Tujuan kajian ini adalah untuk merekodkan kepelbagaian spesies burung di tapak cadangan Stesen Kajiluar Marin UM. Kajian lapangan telah dijalankan sebanyak lima kali sepanjang tempoh 12 bulan dari Jun 2008 hingga Mei 2009. Kaedah pemerhatian langsung telah digunakan bagi merekod spesies burung. Sebanyak 70 spesies burung dari 30 famili telah direkodkan di kawasan kajian. Daripada jumlah ini, tiga spesies adalah diperkenalkan, 45 adalah residen, 11 adalah migran, dan 11 spesies mempunyai kedua-dua populasi migran dan residen. Empat puluh lapan spesies yang direkodkan adalah samada diperlindungi sepenuhnya atau diperlindungi (dua spesies sebagai burung buruan dan dua spesies lain sebagai burung peliharaan berlesen) di bawah Akta Perlindungan Hidupan Liar 1972 (Act 1976). Dari segi status pemuliharaan, dua spesies (Rimba Dahan, Malacopteron albogulare dan Bayan Nuri, Psittacula longicauda) dikelaskan sebagai hampir terancam oleh Buku Data Merah IUCN. Lima puluh dua spesies yang direkodkan biasa ditemui di serata Semenanjung Malaysia tetapi sepuluh spesies tidak biasa ditemui, tujuh adalah melimpah, dan satu spesies (Pucung Jawa, Ardeola speciosa) jarang ditemui. Taburan burung dan kepelbagaian spesies yang direkodkan pada setiap lawatan adalah berbeza bagi setiap tapak kajian. Secara umumnya, daerah Bachok mempunyai varieti burung yang pelbagai dan berupaya menyumbang kepada pemuliharaan dan pengurusan secara signifikan.

(Keywords: bird diversity, bird survey, rural area, biodiversity inventory)

\section{INTRODUCTION}

Malaysia is located within one of the richest biogeographical regions both in terms of species and habitat richness. The avifauna for example is particularly rich. There are 650 species of birds recorded in Peninsular Malaysia and Singapore, some of which $(22 \%)$ are migrants while most species $(62 \%)$ breed locally $[1,2]$. Like other tropical countries, possessing a wide variety of habitats (e.g. lowland mixed-dipterocarp tropical rainforest) with plentiful resources allow Malaysia to harbour a high number of bird species [3]. Malaysian lowland rainforests for example are home to some 311 species of birds (274 are residents), and as in other tropical areas, they are important habitats for biodiversity conservation [4]. Other habitats such as mangrove forest and coastal areas provide resources for migratory birds as they have been using these areas as stopover sites before continuing their journey [5]. Therefore, adequate information on bird distribution in any area of interest is necessary to facilitate biodiversity management and conservation.

Many studies on bird species richness conducted in Peninsular Malaysia have focussed on lowland forests $[6,7,8]$ and islands $[9,10,11]$, with only a few on degraded habitats such as agricultural or rural 
areas $[12,13]$. This is due to the fact that lowland rainforests harbour more bird species than degraded habitats [14], which lead to greater understanding on the important role played by lowland rainforests in bird conservation. It is undeniable that the existence of forested areas is very important for bird conservation but research on degraded habitats should not be neglected. This is because although virgin forests generally have better bird species richness and composition, degraded habitats may still have some pivotal roles to play in terms of biodiversity conservation $[12,13]$. Furthermore, it is important to address the role of degraded forests in supporting bird communities as human population continues to increase and intensification of human land use escalates [15]. Gathering information on bird species inhabiting degraded habitat will eventually lead to better understanding on roles and function played by this habitat $[16,17,18]$. As suggested by Peh et al. [12], degraded habitats and landscapes can, in some cases, serve as surrogate habitats for some of the forest biota. This study was designed to elucidate the contribution of degraded habitats in Bachok district, Kelantan, Peninsular Malaysia towards bird conservation and management. The objective can be achieved by documenting bird species richness in the area and compare it with previous studies in degraded habitats.

\section{MATERIALS AND METHODS}

District of Bachok, Kelantan, Peninsular Malaysia is a rural area located along South China Sea and has a stretch of beautiful beaches. The district is dominated by traditional villages and other human settlements areas interspersed with agricultural areas. There is no forested area within the district but the adjacent district (Pasir Puteh) has few forest reserves. To facilitate the bird survey process, eight study sites (identified as site A to site $\mathrm{H}$ ) with different physical characteristics were established within the study area (Table 1). It is assumed that different physical characteristics will form diverse habitats with a variety of resources that possibly will influence the presence of bird species [19]. Among the habitats available within the study sites were beaches, open grazing field, small and large trees, shrubs, and electrical pylons with cables.

A total of five visits (17-19 June 2008, 15-19 January 2009, 4-8 February 2009, 10-13 March 2009, and 1215 May 2009) were conducted to all study sites within the study area. All surveys were conducted in fair weather (without strong winds and precipitation). During each survey, a direct observation method (using binoculars with $8 \times 40$ magnifications) was used to record bird species diversity. Morning observation session was started at 0730 hours until 1200 hours while afternoon observation session started at 1400 hours until 1830 hours. Each site was visited for 30 minutes (one observation sub-session) to record bird species richness before moving to the next study site. Available tracks within study site (approximately 100 meters long) were used as transects to facilitate observation. Any bird seen or heard within $25-\mathrm{m}$ radius was recorded. Doubtful sightings were confirmed by repeated observations involving note-taking and drawings which later were identified using various field guides $[1,2,20]$. Each site was visited twice daily (two observation subsessions; one each in the morning and afternoon sessions) and a proper schedule was established to ensure all sites were visited at different sub-sessions. All observed birds were identified up to species level and secondary information related to each species such as distribution status, protection status, degree of occurrence, and conservation status (according to IUCN Red List 2001) was referred from published materials $[1,21,22,23]$.

\section{RESULTS}

A total of 70 bird species belonging to 30 families were recorded in the study area during 360 observation hours (Table 2 and Figure 1). Most species are residents (64\% or 45 species), followed by migrants ( $16 \%$ or 11 species), and introduced bird (4\% or three species), while 11 species (or $16 \%$ ) were represented by both resident and migrant populations. The introduced species are White-vented Myna (Acridotheres javanicus), House Crow (Corvus splendens), and Javan Munia (Lonchura leucogastroides). The latter species, observed only at site $G$, is not officially listed in the checklist of Peninsular Malaysia's birds, and is possibly an escapee since it is a popular cage bird.

Seven species $(10 \%)$ present in the study area have been classified as abundantly distributed throughout Peninsular Malaysia. These are White-vented Myna, House Crow, Common Myna (Acridotheres tristis), Eurasian Tree Sparrow (Passer montanus), Yellowvented Bulbul (Pycnonotus goaivier), White-breasted Waterhen (Amaurornis phoenicurus), and Brahminy Kite (Haliastur indus). However, some of these species were not abundantly distributed in the study area. For example, Eurasian Tree Sparrow was observed in site B and $\mathrm{H}$ only while White-breasted Waterhen was recorded in site $\mathrm{G}$ and $\mathrm{H}$ only. The other two regionally abundant species were observed 
Table 1. Location of all study sites and its descriptions

\begin{tabular}{|c|c|c|c|c|}
\hline Site & \multicolumn{2}{|l|}{ Site Descriptions } & Latitude & Long \\
\hline SITE A & \multicolumn{2}{|c|}{$\begin{array}{l}\text { Shrubby areas with small trees; some open grazing } \\
\text { fields }\end{array}$} & N $05^{\circ} 57.433^{\prime}$ & E $102^{\circ} 26.628^{\prime}$ \\
\hline SITE B & \multicolumn{2}{|c|}{$\begin{array}{l}\text { Shrubby areas with small trees; close to beach; some } \\
\text { open grazing fields }\end{array}$} & N $05^{\circ} 57.499^{\prime}$ & E $102^{\circ} 26.277^{\prime}$ \\
\hline SITE C & \multicolumn{2}{|c|}{$\begin{array}{l}\text { Few tall trees with sparse shrub; electrical cables and } \\
\text { pylons }\end{array}$} & N $05^{\circ} 59.247^{\prime}$ & E $102^{\circ} 26.058^{\prime}$ \\
\hline SITE D & \multicolumn{2}{|c|}{$\begin{array}{l}\text { Open area with few tall trees; natural well; grazing } \\
\text { fields }\end{array}$} & N $05^{\circ} 59.434^{\prime}$ & E $102^{\circ} 25.537^{\prime}$ \\
\hline SITE E & \multicolumn{2}{|c|}{$\begin{array}{l}\text { Open/grassy areas with electrical pylon; absence of } \\
\text { taller trees }\end{array}$} & N $05^{\circ} 59.136^{\prime}$ & E $102^{\circ} 25.422^{\prime}$ \\
\hline SITE F & \multicolumn{2}{|c|}{$\begin{array}{l}\text { Swampy area with freshwater supply in small stream } \\
\text { and concrete drain }\end{array}$} & N $05^{\circ} 59.390^{\prime}$ & E $102^{\circ} 25.306^{\prime}$ \\
\hline SITE G & \multicolumn{2}{|c|}{$\begin{array}{l}\text { Open/grassy areas; few small trees; open grazing } \\
\text { fields }\end{array}$} & $\mathrm{N} 05^{\circ} 59.661^{\prime}$ & E $102^{\circ} 25.188^{\prime}$ \\
\hline SITE H & \multicolumn{2}{|c|}{$\begin{array}{l}\text { Open areas with lot of coconut trees; adjacent to } \\
\text { beach }\end{array}$} & $\mathrm{N} 06^{\circ} 00.417^{\prime}$ & E $102^{\circ} 25.583^{\prime}$ \\
\hline \multicolumn{2}{|c|}{$\begin{array}{l}\text { in five study sites only. The House Crow for example } \\
\text { was absent from sites D, F and G while Brahminy } \\
\text { Kite was not recorded in sites A, B and D. On the } \\
\text { other hand, the Javan Pond-Heron (Ardeola speciosa) } \\
\text { maintained its rarity been present only in a single } \\
\text { study site (site F). Another ten species (14\%) } \\
\text { recorded in the study area have been classified as } \\
\text { uncommon throughout Peninsular Malaysia (Table } \\
\text { 2). Most of these species were also uncommon in the } \\
\text { study area (been observed in less than any four study } \\
\text { sites) except Purple-throated Sunbird (Nectarinia } \\
\text { sperata) that was commonly found in the study area } \\
\text { (presence in all sites except sites D and H). } \\
\text { However, some species that are commonly found } \\
\text { throughout Peninsular Malaysia had different } \\
\text { distribution patterns in the study area. For example, }\end{array}$} & \multicolumn{3}{|c|}{$\begin{array}{l}\text { while } 18 \text { species }(26 \%) \text { are not protected by } \\
\text { Protection of Wildlife Act } 1976 \text {. Two species ( } 3 \% \text { ), } \\
\text { the Barred Buttonquail (Turnix suscitator) and } \\
\text { White-breasted Waterhen, are partially protected as } \\
\text { game birds (i.e. they can be hunted under license } \\
\text { from Department of Wildlife and National Parks) and } \\
\text { were found in any two study sites only. The former } \\
\text { species was recorded in sites B and G only while the } \\
\text { latter species was observed in sites G and H only. } \\
\text { Another two species (3\%), Long-tailed Parakeet } \\
\text { (Psittacula longicauda) and Japanese Sparrowhawk } \\
\text { are classified as other protected birds (may be reared } \\
\text { in captivity under licence from Department of } \\
\text { Wildlife and National Parks). Both species have } \\
\text { limited distribution in the study area, found only in } \\
\text { single study site (Long-tailed Parakeet was recorded } \\
\text { in site A only while Japanese Sparrowhawk was } \\
\text { observed in site F only). }\end{array}$} \\
\hline
\end{tabular}
eight species are commonly found in the study area (recorded in any 4 to 6 study sites) while other birds either had a distribution limited to less than any four study sites (37 species) or abundantly present in the area (seven species). Twenty-one of nationally common species such as Japanese Sparrowhawk (Accipiter gularis), Common Kingfisher (Todiramphus chloris), Chinese Pond-Heron (Ardeola bacchus), Ashy Minivet (Pericrocotus divaricatus), Brown Shrike (Lanius cristatus), Crested Serpent Eagle (Spilornis cheela), Yellow Wagtail (Motacilla flava), etc. are rarely found in the study area (they were recorded in any single study site only).

In term of protection status, most of the recorded species (48 species or $68 \%$ ) are totally protected

Species composition varied from one site to another. Site D had the least number of species (14 species) while site B had most species (35 species). Another two sites that had less species number were sites $C$ and E. Both sites had 20 and 21 species respectively. Other sites had moderate number of species that ranged from 29 to 32 species (Figure 2). Although most birds (26 species or $37 \%$ ) were observed at any single site only, eight species $(12 \%)$ were observed at all study sites while two species (3\%) were recorded present in any seven sites. Species that were recorded in all sites were Asian Koel (Eudynamys scolopacea), Common Myna, Glossy Swiftlet (Collocalia esculenta), House Swift (Apus affinis), Pacific Swallow (Hirundo tahitica), Spotted Dove (Streptopelia chinensis), White-vented Myna, and Yellow-vented Bulbul (Pycnonotus goaivier). Two species that were recorded from any seven sites were 
Table 2. List of species recorded in study area with distribution status, protection status, and degree of occurrence.

\begin{tabular}{|c|c|c|c|c|c|}
\hline No. & Species & Family & $\begin{array}{l}\text { Distribution } \\
\text { Status }\end{array}$ & $\begin{array}{l}\text { Protection } \\
\text { Status }\end{array}$ & $\begin{array}{l}\text { Degree of } \\
\text { Occurrence }\end{array}$ \\
\hline 1 & $\begin{array}{l}\text { Japanese Sparrowhawk } \\
\text { Accipiter gularis }\end{array}$ & Accipitridae & M & OPB & $\mathrm{C}$ \\
\hline 2 & $\begin{array}{l}\text { White-vented Myna } \\
\text { Acridotheres javanicus }\end{array}$ & Sturnidae & I & NP & A \\
\hline 3 & $\begin{array}{l}\text { Common Myna Acridotheres } \\
\text { tristis }\end{array}$ & Sturnidae & $\mathrm{R}$ & NP & A \\
\hline 4 & Common Iora Aegithina tiphia & Chloropseidae & $\mathrm{R}$ & $\mathrm{TP}$ & $\mathrm{C}$ \\
\hline 5 & $\begin{array}{l}\text { Common Kingfisher Alcedo } \\
\text { atthis }\end{array}$ & Alcedinidae & $\mathrm{R} \& \mathrm{M}$ & $\mathrm{TP}$ & $\mathrm{C}$ \\
\hline 6 & $\begin{array}{l}\text { White-breasted Waterhen } \\
\text { Amaurornis phoenicurus }\end{array}$ & Rallidae & $\mathrm{R} \& \mathrm{M}$ & GB & $\mathrm{A}$ \\
\hline 7 & $\begin{array}{l}\text { Brown-throated Sunbird } \\
\text { Anthreptes malaccensis }\end{array}$ & Nectariniidae & $\mathrm{R}$ & $\mathrm{TP}$ & $\mathrm{C}$ \\
\hline 8 & Richard's Pipit Anthus richardi & Motacillidae & $\mathrm{R} \& \mathrm{M}$ & NP & $\mathrm{C}$ \\
\hline 9 & House Swift Apus affinis & Apodidae & $\mathrm{R}$ & $\mathrm{TP}$ & $\mathrm{C}$ \\
\hline 10 & $\begin{array}{l}\text { Chinese Pond-Heron Ardeola } \\
\text { bacchus }\end{array}$ & Ardeidae & M & $\mathrm{TP}$ & $\mathrm{C}$ \\
\hline 11 & $\begin{array}{l}\text { Javan Pond-Heron Ardeola } \\
\text { speciosa }\end{array}$ & Ardeidae & M & NP & RA \\
\hline 12 & Cattle Egret Bubulcus ibis & Ardeidae & $\mathrm{R} \& \mathrm{M}$ & $\mathrm{TP}$ & $\mathrm{C}$ \\
\hline 13 & $\begin{array}{l}\text { Grey-faced Buzzard Butastur } \\
\text { indicus }\end{array}$ & Accipitridae & M & $\mathrm{TP}$ & $\mathrm{C}$ \\
\hline 14 & $\begin{array}{l}\text { Rufous Woodpecker Celeus } \\
\text { brachyurus }\end{array}$ & Picidae & $\mathrm{R}$ & $\mathrm{TP}$ & $\mathrm{C}$ \\
\hline 15 & $\begin{array}{l}\text { Lesser Coucal Centropus } \\
\text { bengalensis }\end{array}$ & Cuculidae & $\mathrm{R}$ & TP & $\mathrm{C}$ \\
\hline 16 & $\begin{array}{l}\text { Greater Coucal Centropus } \\
\text { sinensis }\end{array}$ & Cuculidae & $\mathrm{R}$ & $\mathrm{TP}$ & $\mathrm{C}$ \\
\hline 17 & $\begin{array}{l}\text { Greater Flameback } \\
\text { Chrysocolaptes lucidus }\end{array}$ & Picidae & $\mathrm{R}$ & $\mathrm{TP}$ & $\mathrm{C}$ \\
\hline 18 & $\begin{array}{l}\text { White-bellied Swiftlet } \\
\text { Collocalia esculenta }\end{array}$ & Apodidae & $\mathrm{R}$ & $\mathrm{TP}$ & $\mathrm{C}$ \\
\hline 19 & $\begin{array}{l}\text { Oriental Magpie Robin } \\
\text { Copsychus saularis }\end{array}$ & Turdidae & $\mathrm{R}$ & NP & $\mathrm{C}$ \\
\hline 20 & $\begin{array}{l}\text { Indian Roller Coracias } \\
\text { benghalensis }\end{array}$ & Coraciidae & $\mathrm{R}$ & $\mathrm{TP}$ & $\mathrm{UC}$ \\
\hline 21 & House Crow Corvus splendens & Corvidae & I & NP & A \\
\hline 22 & $\begin{array}{l}\text { Scarlet-backed Flowerpecker } \\
\text { Dicaeum cruentatum }\end{array}$ & Dicaeidae & $\mathrm{R}$ & $\mathrm{TP}$ & $\mathrm{C}$ \\
\hline 23 & $\begin{array}{l}\text { Crow-billed Drongo Dicrurus } \\
\text { annectans }\end{array}$ & Dicruridae & $\mathrm{M}$ & $\mathrm{TP}$ & $\mathrm{UC}$ \\
\hline 24 & $\begin{array}{l}\text { Black Drongo Dicrurus } \\
\text { macrocercus }\end{array}$ & Dicruridae & M & $\mathrm{TP}$ & $\mathrm{C}$ \\
\hline 25 & $\begin{array}{l}\text { Greater Racket-Tailed Drongo } \\
\text { Dicrurus paradiseus }\end{array}$ & Dicruridae & $\mathrm{R}$ & $\mathrm{TP}$ & $\mathrm{C}$ \\
\hline 26 & $\begin{array}{l}\text { Common Flameback Dinopium } \\
\text { javanense }\end{array}$ & Picidae & $\mathrm{R}$ & $\mathrm{TP}$ & $\mathrm{C}$ \\
\hline 27 & $\begin{array}{l}\text { Common Koel Eudynamys } \\
\text { scolopacea }\end{array}$ & Cuculidae & $\mathrm{R} \& \mathrm{M}$ & $\mathrm{TP}$ & $\mathrm{C}$ \\
\hline
\end{tabular}




\begin{tabular}{|c|c|c|c|c|c|}
\hline No. & Species & Family & $\begin{array}{c}\text { Distribution } \\
\text { Status }\end{array}$ & $\begin{array}{l}\text { Protection } \\
\text { Status }\end{array}$ & $\begin{array}{c}\text { Degree of } \\
\text { Occurrence }\end{array}$ \\
\hline 28 & $\begin{array}{l}\text { Dollarbird Eurystomus } \\
\text { orientalis }\end{array}$ & Coraciidae & $\mathrm{R} \& \mathrm{M}$ & $\mathrm{TP}$ & $\mathrm{C}$ \\
\hline 29 & Peaceful Dove Geopelia striata & Columbidae & $\mathrm{R}$ & NP & $\mathrm{C}$ \\
\hline 30 & $\begin{array}{l}\text { White-throated Kingfisher } \\
\text { Halcyon smyrnensis }\end{array}$ & Alcedinidae & $\mathrm{R}$ & $\mathrm{TP}$ & $\mathrm{C}$ \\
\hline 31 & Brahminy Kite Haliastur indus & Accipitridae & $\mathrm{R}$ & $\mathrm{TP}$ & A \\
\hline 32 & $\begin{array}{l}\text { Pacific Swallow Hirundo } \\
\text { tahitica }\end{array}$ & Hirundinidae & $\mathrm{R}$ & TP & $\mathrm{C}$ \\
\hline 33 & $\begin{array}{l}\text { Cinnamon Bittern Ixobrychus } \\
\text { cinnamomeus }\end{array}$ & Ardeidae & $\mathrm{R} \& \mathrm{M}$ & ТP & $\mathrm{C}$ \\
\hline 34 & Pied Triller Lalage nigra & Campephagidae & $\mathrm{R}$ & $\mathrm{TP}$ & $\mathrm{C}$ \\
\hline 35 & Brown Shrike Lanius cristatus & Laniidae & M & $\mathrm{TP}$ & $\mathrm{C}$ \\
\hline 36 & Tiger Shrike Lanius tigrinus & Laniidae & M & $\mathrm{TP}$ & $\mathrm{C}$ \\
\hline 37 & $\begin{array}{l}\text { White-bellied Munia Lonchura } \\
\text { leucogastra }\end{array}$ & Estrildidae & $\mathrm{R}$ & NP & $\mathrm{UC}$ \\
\hline 38 & $\begin{array}{l}\text { Javan Munia Lonchura } \\
\text { leucogastroides }\end{array}$ & Estrildidae & I & NP & $\mathrm{C}$ \\
\hline 39 & $\begin{array}{l}\text { White-headed Munia Lonchura } \\
\text { maja }\end{array}$ & Estrildidae & $\mathrm{R}$ & NP & $\mathrm{C}$ \\
\hline 40 & $\begin{array}{l}\text { Scaly-breasted Munia } \\
\text { Lonchura punctulata }\end{array}$ & Estrildidae & $\mathrm{R}$ & NP & $\mathrm{C}$ \\
\hline 41 & $\begin{array}{l}\text { White-rumped Munia } \\
\text { Lonchura striata }\end{array}$ & Estrildidae & $\mathrm{R}$ & NP & $\mathrm{UC}$ \\
\hline 42 & $\begin{array}{l}\text { Grey-breasted Babbler } \\
\text { Malacopteron albogulare }\end{array}$ & Timaliidae & $\mathrm{R}$ & ТP & $\mathrm{UC}$ \\
\hline 43 & $\begin{array}{l}\text { Lineated Barbet Megalaima } \\
\text { lineata }\end{array}$ & Capitonidae & $\mathrm{R}$ & $\mathrm{TP}$ & $\mathrm{C}$ \\
\hline 44 & $\begin{array}{l}\text { Chestnut-headed Bee-eater } \\
\text { Merops leschenaulti }\end{array}$ & Meropidae & $\mathrm{R}$ & $\mathrm{TP}$ & $\mathrm{UC}$ \\
\hline 45 & $\begin{array}{l}\text { Blue-tailed Bee-eater Merops } \\
\text { philippinus }\end{array}$ & Meropidae & $\mathrm{R} \& \mathrm{M}$ & NP & $\mathrm{C}$ \\
\hline 46 & $\begin{array}{l}\text { Blue-throated Bee-eater } \\
\text { Merops viridis }\end{array}$ & Meropidae & $\mathrm{R} \& \mathrm{M}$ & TP & $\mathrm{C}$ \\
\hline 47 & Yellow Wagtail Motacilla flava & Motacillidae & M & TP & $\mathrm{C}$ \\
\hline 48 & $\begin{array}{l}\text { Olive-backed Sunbird } \\
\text { Nectarinia jugularis }\end{array}$ & Nectariniidae & $\mathrm{R}$ & $\mathrm{TP}$ & $\mathrm{C}$ \\
\hline 49 & $\begin{array}{l}\text { Purple-throated Sunbird } \\
\text { Nectarinia sperata }\end{array}$ & Nectariniidae & $\mathrm{R}$ & $\mathrm{TP}$ & $\mathrm{UC}$ \\
\hline 50 & $\begin{array}{l}\text { Black-naped Oriole Oriolus } \\
\text { chinensis }\end{array}$ & Oriolidae & $\mathrm{R} \& \mathrm{M}$ & TP & $\mathrm{C}$ \\
\hline 51 & $\begin{array}{l}\text { Dark-necked Tailorbird } \\
\text { Orthotomus atrogularis }\end{array}$ & Sylviidae & $\mathrm{R}$ & $\mathrm{TP}$ & $\mathrm{C}$ \\
\hline 52 & $\begin{array}{l}\text { Ashy Tailorbird Orthotomus } \\
\text { sepium }\end{array}$ & Sylviidae & $\mathrm{R}$ & $\mathrm{TP}$ & $\mathrm{C}$ \\
\hline 53 & $\begin{array}{l}\text { Rufous-tailed Tailorbird } \\
\text { Orthotomus sericeus }\end{array}$ & Sylviidae & $\mathrm{R}$ & $\mathrm{TP}$ & $\mathrm{UC}$ \\
\hline 54 & $\begin{array}{l}\text { Common Tailorbird } \\
\text { Orthotomus sutorius }\end{array}$ & Sylviidae & $\mathrm{R}$ & $\mathrm{TP}$ & $\mathrm{C}$ \\
\hline 55 & $\begin{array}{l}\text { Plain-backed sparrow Passer } \\
\text { flaveolus }\end{array}$ & Ploceidae & $\mathrm{R}$ & NP & UC \\
\hline
\end{tabular}




\begin{tabular}{|c|c|c|c|c|c|}
\hline No. & Species & Family & $\begin{array}{c}\text { Distribution } \\
\text { Status }\end{array}$ & $\begin{array}{l}\text { Protection } \\
\text { Status } \\
\end{array}$ & $\begin{array}{c}\text { Degree of } \\
\text { Occurrence }\end{array}$ \\
\hline 56 & $\begin{array}{l}\text { Eurasian Tree Sparrow Passer } \\
\text { montanus }\end{array}$ & Ploceidae & $\mathrm{R}$ & NP & A \\
\hline 57 & $\begin{array}{l}\text { Ashy Minivet Pericrocotus } \\
\text { divaricatus }\end{array}$ & Campephagidae & M & TP & $\mathrm{C}$ \\
\hline 58 & $\begin{array}{l}\text { Chestnut-breasted Malkoha } \\
\text { Phaenicophaeus curvirostris }\end{array}$ & Cuculidae & $\mathrm{R}$ & NP & $\mathrm{C}$ \\
\hline 59 & $\begin{array}{l}\text { Green-billed Malkoha } \\
\text { Phaenicophaeus tristis }\end{array}$ & Cuculidae & $\mathrm{R}$ & $\mathrm{TP}$ & $\mathrm{C}$ \\
\hline 60 & $\begin{array}{l}\text { Banded Woodpecker Picus } \\
\text { miniaceus }\end{array}$ & Picidae & $\mathrm{R}$ & $\mathrm{TP}$ & $\mathrm{C}$ \\
\hline 61 & $\begin{array}{l}\text { Crimson breasted Flowerpecker } \\
\text { Prionochilus percussus }\end{array}$ & Dicaeidae & $\mathrm{R}$ & $\mathrm{TP}$ & $\mathrm{C}$ \\
\hline 62 & $\begin{array}{l}\text { Long-tailed Parakeet Psittacula } \\
\text { longicauda }\end{array}$ & Psittacidae & $\mathrm{R}$ & OPB & $\mathrm{C}$ \\
\hline 63 & $\begin{array}{l}\text { Stripe-throated Bulbul } \\
\text { Pycnonotus finlaysoni }\end{array}$ & Pycnonotidae & $\mathrm{R}$ & $\mathrm{TP}$ & $\mathrm{C}$ \\
\hline 64 & $\begin{array}{l}\text { Yellow-vented Bulbul } \\
\text { Pycnonotus goaivier }\end{array}$ & Pycnonotidae & $\mathrm{R}$ & NP & A \\
\hline 65 & $\begin{array}{l}\text { Pied Fantail Rhipidura } \\
\text { javanica }\end{array}$ & Rhipiduridae & $\mathrm{R}$ & $\mathrm{TP}$ & $\mathrm{C}$ \\
\hline 66 & $\begin{array}{l}\text { Crested Serpent Eagle Spilornis } \\
\text { cheela }\end{array}$ & Accipitridae & $\mathrm{R}$ & $\mathrm{TP}$ & $\mathrm{C}$ \\
\hline 67 & $\begin{array}{l}\text { Rufous-fronted Babbler } \\
\text { Stachyris rufifrons }\end{array}$ & Timaliidae & $\mathrm{R}$ & ТP & UC \\
\hline 68 & $\begin{array}{l}\text { Spotted Dove Streptopelia } \\
\text { chinensis }\end{array}$ & Columbidae & $\mathrm{R}$ & NP & $\mathrm{C}$ \\
\hline 69 & $\begin{array}{l}\text { Collared Kingfisher } \\
\text { Todiramphus chloris }\end{array}$ & Alcedinidae & $\mathrm{R} \& \mathrm{M}$ & $\mathrm{TP}$ & $\mathrm{C}$ \\
\hline 70 & $\begin{array}{l}\text { Barred Buttonquail Turnix } \\
\text { suscitator }\end{array}$ & Turnicidae & M & GB & $\mathrm{C}$ \\
\hline
\end{tabular}

Legend: Distribution status ( $\mathrm{I}=$ Introduced, $\mathrm{R}=$ Resident, $\mathrm{M}=$ Migrant); Protection Status $(\mathrm{NP}=$ Not protected, $\mathrm{TP}$ $=$ Totally protected, $\mathrm{GB}=$ Game birds, $\mathrm{OPB}=$ Other protected birds $)$; Degree of occurrence $(\mathrm{A}=$ Abundant, $\mathrm{C}=$ Common, $\mathrm{UC}=$ Uncommon, $\mathrm{RA}=$ Rare).

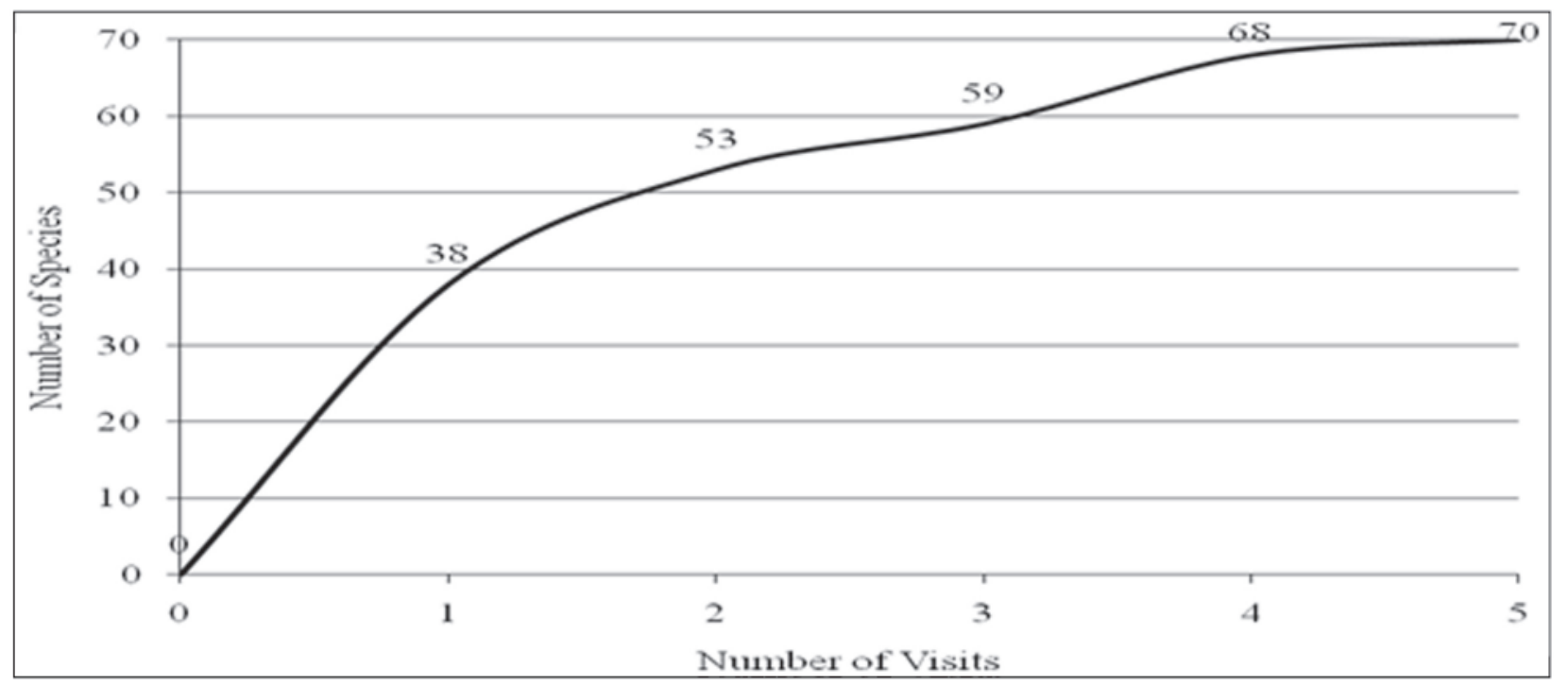

Figure 1. Species accumulation curve for bird population in Bachok coastal areas 


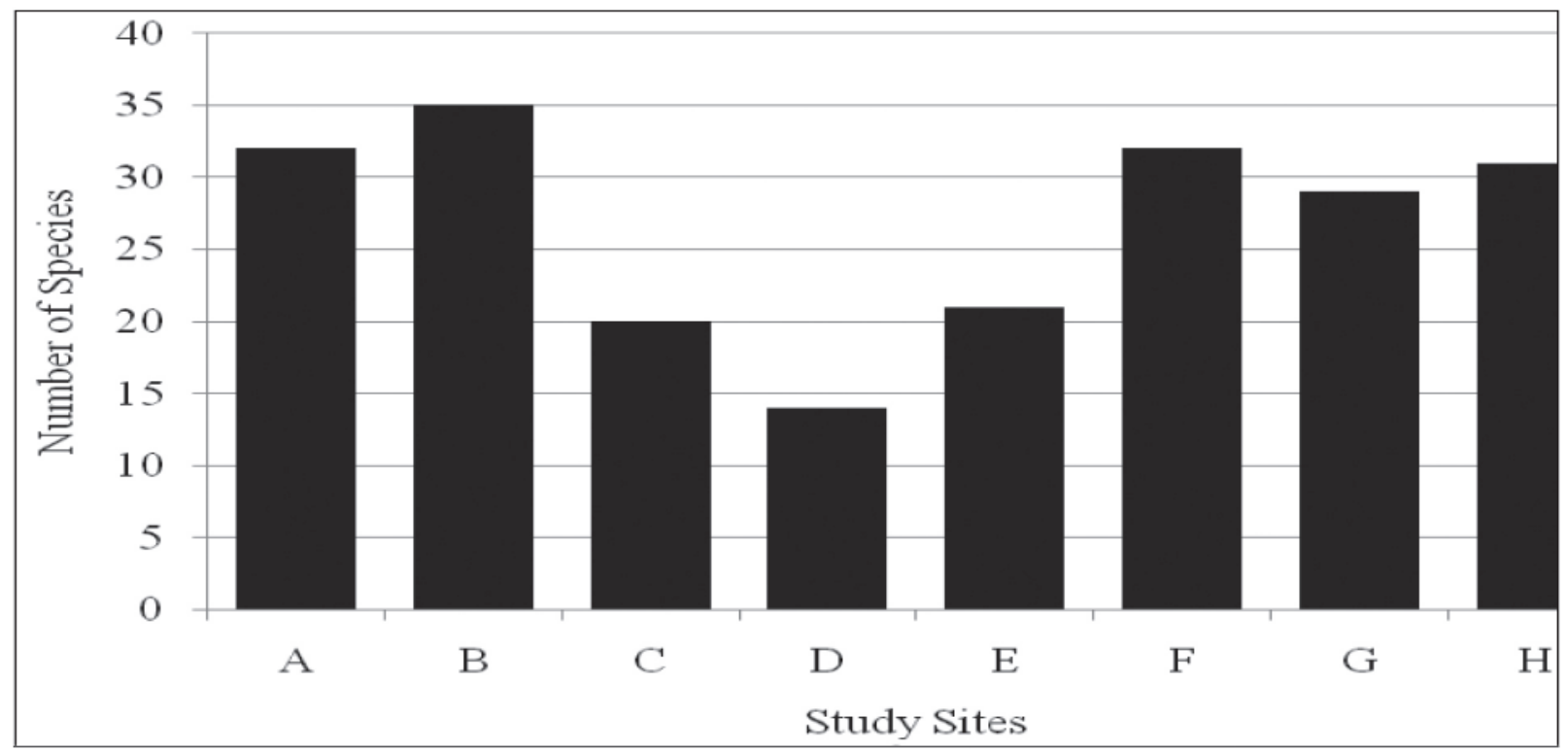

Figure 2. Number of bird species recorded in each study site

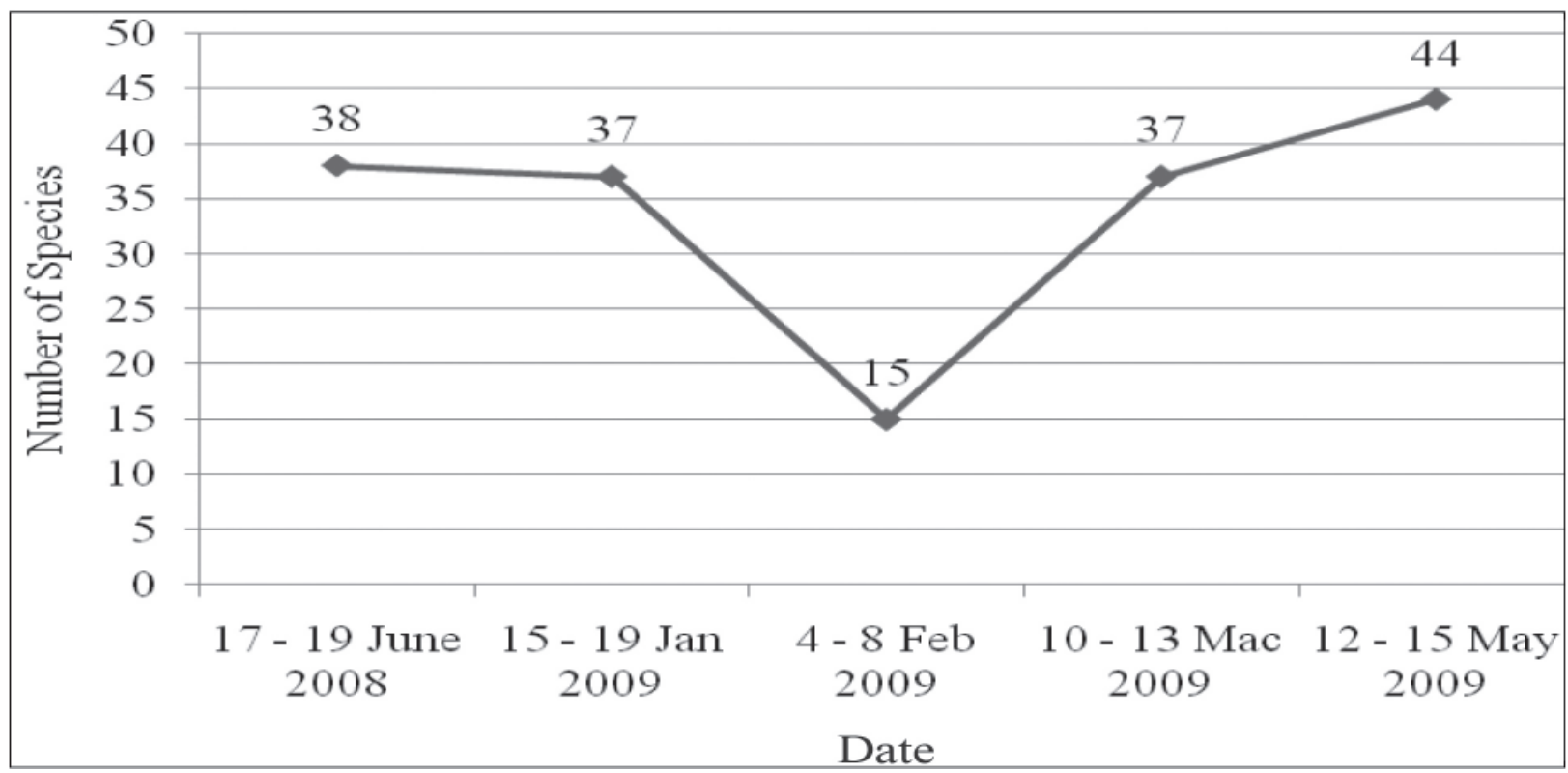

Figure 3. Total number of species recorded during each visit

Peaceful Dove (Geopelia striata) and White-throated Kingfisher (Halcyon smyrnensis). Thirteen species $(19 \%)$ were recorded from any two sites, ten species (14\%) were observed in any three sites, and between three to five species $(4-7 \%)$ were observed either in any four, five or six sites.

Visitation date also contributed to the difference in number of species recorded. More species were recorded during the May 2009 visit (44 species or $26 \%$ ) compared to February 2009 visit (14 species or $9 \%$ ). The other three visits recorded either 37 or 38 species (Figure 3). In term of visit frequency, none of the bird species were repeatedly observed in all five visits. A total of 21 species (30\%) were recorded in any single visit only or during any four visits, 17 species $(24 \%)$ were recorded during any two visits, and 11 species $(16 \%)$ were observed during any three visits.

\section{DISCUSSIONS}

The study shows that Bachok district had quite a number of bird species that have adapted well as indicated by the presence of at least 70 species of 
birds in human dominated habitat. The presence of three introduced species in the study area leads to mixed conclusions. It is well known that Whitevented Myna and House Crow have managed to successfully disperse throughout the country especially in human dominated habitat. Therefore, it is expected that these species will be recorded in the area. However, the presence of Javan Munia is unexpected. This is because the species is not in Peninsular Malaysia bird checklist and do not have widely distributied compared to the two former species. However, there is a possibility that the bird had been released into the wild or escaped from captivity. This is because its population is small, been observed in one study site only.

Although the total number of bird species that were present in the study area is only about ten percent of the total Malaysian bird population [1,2,21] it is still a valuable record when comparing other places. Other degraded habitats such as plantation area in southern Johor [13] had less species than Bachok district, while Carey Island [10] and Gunung Belumut, Johor [12] have almost the same number of bird species as Bachok district. The virgin forest of Mount Siburan, Philippines [19] has a similar number of bird species as Bachok district. However, it is inaccurate to evaluate the capacity of an area to harbour bird species based on the total number of species alone. Species composition should also be used to determine the functional role of any habitat towards bird conservation. Bachok district has a different species composition as compared to the above stated areas. Species recorded from Bachok district are dominated by open country (44 species) or mangrove forest birds ( 24 species) with the least conservation concern. The presence of open habitats such as grazing field and small trees or shrubs in Bachok has apparently attracted many open country birds. However, the presence of mangrove birds is difficult to explain since the nearest patch of mangrove forest is located about five kilometres away. The only possibility is that mangrove species in this area have already adapted to the local conditions to survive even in shrubby area available along the beach. Some species are able to survive in this condition. The presence of forest birds also indicates that even degraded habitats in Bachok are able to provide adequate resources for bird survival [24]. On the other hand, these species may have no choice other than to adapt and survive in the degraded habitat after they lost their original habitats. Unfortunately, no comparison could be made due to the lack of data on bird species richness before habitat modification. Some of the forest's species may have disappeared from the area during the course of modification.

This study indicates that the degree of occurrence can vary at local and regional scales depending on available resources. Even if the species is abundant or commonly found throughout the country, it does not necessarily enjoy the same scale of distribution at the local scale. Two species that were recorded in the study area, Eurasian Tree Sparrow and Whitebreasted Waterhen are good examples to explain this phenomenon. The former requires grassy areas with seeds as food resources, while the latter is a generalist that frequents watery areas that have seeds and insects. Only a few study sites within the study area fulfil the requirements to support these birds. Thus, sites that do not have these resources are unable to attract the sparrow and waterhen. Nationally common species such as Japanese Sparrowhawk, Common Kingfisher, Chinese PondHeron, etc. also have limited distribution in the study area, possibly due to unavailability of food or nesting resources to support larger populations.

It is expected to record more common and nonprotected species in Bachok district since it is human dominated habitat. However, the presence of two game birds (Barred Buttonquail and White-breasted Waterhen) and two cage birds (Long-tailed Parakeet and Japanese Sparrowhawk) beg further explanation. Furthermore, Long-tailed Parakeet is listed as Nearly Threatened species [21]. All four species have been recorded in less than three study sites, indicating their rarity within the study area. Their small population may reflect hunting pressure (they can be hunted with permission from Department of Wildlife and National Parks either as game or cage birds). Otherwise, the presence of Long-tailed Parakeet may also be due to escape from captivity. Whatever the reason may be, more attention should be paid to these four species since their distributions are limited in the study area (Long-tailed Parakeet was observed in site A only, while Japanese Sparrowhawk was recorded in site $\mathrm{F}$ only). One way to improve the quality of degraded habitats like Bachok for biodiversity is to plant more shrubs and trees, improve water restoration process, and increase vegetation diversity. These actions not only improve bird diversity in the area but also improve the quality of human and wildlife $[17,25]$.

Variation in species composition in each study site is mainly caused by the availability of habitat and food resources in the area [24]. Sites B and $\mathrm{H}$ for example were located closer to the beach and had a shrubby area that resembled a mangrove forest patch. This 
allows the sites to harbour mangrove species such as Collared Kingfisher and Banded Woodpecker (Picus miniaceus). On the other hand, Cinnamon Bittern (Ixobrychus cinnamomeus) and Javan Pond-Heron were only present in site $\mathrm{F}$ since the area had a freshwater water body that provided a suitable habitat for them. Sites E and G were grassy open areas that attracted granivorous species such as Plain-backed Sparrow (Passer flaveolus) and White-headed Munia (Lonchura maja).

Due to seasonal differences, different species were recorded during different visits. Two main seasons that influence the results were the migratory and wetdry seasons. Migratory species will only be recorded during migratory season which is from mid October until May. Therefore, any visit falling within this period will see more migratory species than local species. Some migratory species compete for similar resources with the local species and may temporarily displaced them from the area, resulting in a decline of their population [5]. Examples of resident species with dwindling populations during the migratory season include Chestnut-breasted Malkoha (Phaenicophaeus curvirostris), Blue-tailed Bee-eater (Merops philippinus), and Rufous Woodpecker (Celeus brachyurus). More birds were observed during the May visit due to the influx of migratory species into the study area. Some migrants used the site as final destination, whereas others use it as a transit or stop-over site for refuelling. The wet-dry seasons indirectly influence bird populations through their effect on food resources and suitability of habitat. The population of birds such as WhiteBreasted Waterhen, Chinese-Pond Heron, and Cinnamon Bittern will increase during the wet season since they are associated with water bodies.

\section{ACKNOWLEDGEMENTS}

We would like to extend our gratitude to the local people of Bachok, Kelantan for their cooperation, assistance and understanding. Our gratitude also goes to many people who had assisted us in the field especially Professor Dr. Mohd Sofian Azirun, Professor Dr. Rosli Hashim, Mr. NorAzhar, and Mr. Khairul Badri. We also would like to thank two anonymous reviewers for their constructive comments to improve the quality of this manuscript. This study was supported by University of Malaya Research Grant No. FS292-2008A awarded to the senior author.

\section{REFERENCES}

1. Strange, M. \& Jeyarajasingam, A. 1993. A Photographic guide to the birds of Peninsular
Malaysia and Singapore. A Sun Tree Notebook, Singapore.

2. Jeyarajasingam, A. \& Pearson, A. 1998. A field guide to the birds of West Malaysia and Singapore. Oxford University Press, Oxford, UK.

3. Sodhi, N.S., Posa, M.R.C., Lee, T.M., Bickford, D., Koh, L.P. \& Brook, B.W. 2009. The state and conservation of Southeast Asian biodiversity. Biodiversity Conservation, On-line first, publishes on-line on $4^{\text {th }}$ March 2009. DOI 10.1007/s10531-009-9607-5.

4. Sodhi, N.S. \& Smith, K.G. 2007. Conservation of tropical birds: mission possible? Journal Ornithology, 148 (Suppl 2): S305-S309.

5. Morris, S.R., Larracuente, A.M., Covino, K.M., Mustillo, M.S., Mattern, K.E., Liebner, D.A. \& Sheets, H.D. 2006. Utility of open population models: limitations posed by parameter estimability in the study of migratory stopover. The Wilson Journal of Ornithology, 118:513526.

6. Wong, M. 1986. Trophic organization of understorey birds in a Malaysian dipterocarp forest. $A u k, 103$ : 100-116.

7. Ramli, R. 2004. Temporal changes in diversity and similarity of bird communities of three forest fragments in an urban environment in Peninsular Malaysia. Malaysian Journal of Science, 23: 8188.

8. Ramli, R., Hashim, R. \& Daicus, B. 2004. Use of mist-netting technique to study community structure of understorey birds at Lubok Tapah Base Camp, Endau-Rompin National Park, Johore, Malaysia. Malaysian Journal of Science, 23: 79-83.

9. Sodhi, N.S., Briffett, C., Lee, B.P.Y-H. \& Subaraj, R. 1999. An annotated checklist of the birds of Pulau Tioman, Peninsular Malaysia. The Raffles Bulletin of Zoology, Supplement No. 6: 125-1330.

10. Ramli, R., Hashim, R., Sofian-Azirun, M. \& Norma-Rashid, Y. 2006. Birds of Carey Island. Golden Hope Plantations and Institute of Biological Sciences, University of Malaya.

11. Ramli, R., Sofian-Azirun, M. \& Hashim, R. 2008. Bird Diversity of three islands in the Straits of Malacca. Malaysian Journal of Science, 27: 129-135.

12. Peh, K.S-H., de Jong, J., Sodhi, N.S., Lim, S.LH. \& Yap, C. A-M. 2005. Lowland rainforest avifauna and human disturbance: persistence of primary forest birds in selectively logged forests and mixed-rural habitats of southern Peninsular Malaysia. Biological Conservation, 123: 489505. 
13 Peh, K. S-H., Sodhi, N. S., de Jong, J., Sekercioglu, C.H., Yap, C.A-M. \& Lim, S.L-H. 2006. Conservation value of degraded habitats for forest birds in southern Peninsular Malaysia. Diversity and Distributions, 12: 572-581.

14. Lambert, F.R. \& Collar, N.J. 2002. The future for Sundaic lowland forest birds: Long-term effects of commercial logging and fragmentation. Forktail 18: 127-146

15. Suarez-Rubio, M. \& Thomlinson, J.R. 2009. Landscape and patch-level factors influence bird communities in an urbanized tropical island. Biological Conservation, 142: 1311-1321.

16. Hughes, J. B., Daily, G. C. \& Ehrlich, P. R. 2002. Conservation of tropical forest birds in countryside habitats. Ecology Letters. 5:121129.

17. Raman, T.R.S \& Sukumar, R. 2002. Responses of tropical rainforest birds to abandoned plantations, edges and logged forest in the Western Ghats, India. Animal Conservation, 5: 201-216.

18. Luck, G. W. \& Daily, G. C. 2003. Tropical countryside bird assemblages: richness, composition, and foraging differ by landscape context. Ecological Applications, 13: 235-247.

19. Lee, D. C. \& Marsden, S. J. 2008. Increasing the value of bird-habitat studies in tropical forests: choice of approach and habitat measures. Bird Conservation International, 18:1-16.

20. Lekagul. B. \& Round, P. D. 1991. A guide to the birds of Thailand. Saha Karn Bhaet Co. Ltd., Bangkok. $457 \mathrm{pp}$.

21. MNS-Bird Conservation Council. 2005. A checklist of the birds of Malaysia - Conservation publication No. 2. Malaysian Nature Society, Kuala Lumpur, Malaysia.

22. Wells, D. R. 1999. The Birds of the Thai-Malay Peninsula.Volume One - Non Passerines. Academic Press.

23. Wells, D. R. 2006. The Birds of the Thai-Malay Peninsula. Volume Two - Passerines. Christopher Helm.

24. Walker, J.S. 2006. Resource use and rarity among frugivorous birds in a tropical rain forest on Sulawesi. Biological Conservation, 130: 6069.

25. Clergeau, P., Jokimäki, J. \& Savard, J-P.L. 2001. Are urban bird communities influenced by the bird diversity of adjacent landscapes. Journal of Applied Ecology, 38: 1122-1134. 